Pacific Journal of Mathematics

A SET OF NONNORMAL NUMBERS 


\title{
A SET OF NONNORMAL NUMBERS
}

\author{
Michel Mendès France
}

Let $P$ be the set of real polynomials and let $E(P)$ be the the set of real numbers whose $n$th binary digit from a certain point on is 0 or 1 according as $[\varphi(n)]$ is even or odd for some $\varphi \in P$. We prove that no number in $E(P)$ is normal in the binary system and that $E(P)$ has Hausdorff dimension 0 .

Some notations and definitions. It is well known that every real number $x$ of the unit interval which is not a binary fraction can be expanded in the binary system

$$
x=\sum_{n=1}^{\infty} \frac{\varepsilon_{n}(x)}{2^{n}}
$$

where $\left(\varepsilon_{n}(x)\right)_{n \in \mathrm{N}}$ is a uniquely determined sequence of functions taking values 0 or 1 . The functions $r_{n}(x)=1-2 \varepsilon_{n}(x)$ are known as the Rademacher functions.

We shall say that $x$ is a normal number (in the binary system) if for every positive integer s and every sequence of positive, strictly increasing integers $k_{1}, k_{2}, \cdots, k_{s}$ one has:

$$
\lim _{N \rightarrow \infty} \frac{1}{N} \sum_{n=1}^{N} r_{n+k_{1}}(x) \cdots r_{n+k_{s}}(x)=0 \text {. }
$$

One can prove that this definition is equivalent to the other usual ones [3], [4], [6].

If $t$ is a real number, [ $t$ ] will denote the greatest integer not greater than $t$ and $\{t\}=t-[t]$ the fractional part of $t$.

Let $P$ be the set of real polynomials and let $E(P)$ be the set of points $x$ such that for some $\varphi \in P$ and for some $n_{0} \geqq 0, r_{n}(x)=$ $\exp i \pi[\varphi(n)]$ for all integers $n>n_{0}$.

We wish to prove first the following theorem:

THEOREM 1. $E(P)$ contains only nonnormal numbers.

This result shows that the measure of $E(P)$ is null, since almost all numbers are normal. Now the question arises if $E(P)$ contains "almost all" (in a sense soon to be made precise) nonnormal numbers or not. We answer this question by stating the known result:

Received July 30, 1964. The preparation of this paper was sponsored by the Office of Naval Research. Reproduction in whole or in part is permitted for any purpose of the United States Government. 
The Hausdorff dimension of the set of nonnormal numbers is 1 , (see for example [1]), and by proving our second theorem :

THEOREM 2. The Hausdorff dimension of $E(P)$ is 0 .

2. Proof of Theorem 1. Let $x$ be an element of $E(P)$ 。 We show that for a certain sequence of increasing positive integers $k_{1}, k_{2}$, $\cdots, k_{s}$ the equation (1) does not hold.

Let $\varphi$ be a polynomial such that $r_{n}(x)=\exp i \pi[\varphi(n)]$ for all sufficiently large integers $n$. Without loss of generality we may suppose that this relation holds for all positive integers, for normality or nonnormality are asymptotic properties. Let the expansion of $\rho$ be

$$
\varphi(n)=\alpha_{\mu \nu} n^{\nu}+\alpha_{\nu-1} n^{\nu-1}+\cdots+\alpha_{1} n+\alpha_{0}, \quad \nu \geqq 1 .
$$

If all the numbers $\alpha_{j}(1 \leqq j \leqq \nu)$ are rational, then $x$ is clearly rational, hence nonnormal. If one of the numbers $\alpha_{j}(1 \leqq j \leqq \nu)$ is irrational, we can without loss of generality suppose that the leading coefficient $\alpha$, is irrational. Indeed, suppose that $\alpha_{\mu}(1 \leqq \mu<\nu)$ is irrational and that $\alpha_{\mu+1}, \cdots, \alpha_{\nu}$ are rational. Let $q$ be the least common denominator of the $\nu-\mu$ fractions $\alpha_{\mu+1} \cdots, \alpha_{\nu}$. If $x$ is normal, then so is the number $y$ defined by $r_{n}(y)=\exp i \pi[\varphi(2 q n)]$ for all integers $n$. But clearly $[\varphi(2 q n)] \equiv[\psi(n)](\bmod 2)$ where $\psi(n)=\alpha_{\mu}(2 q)^{\mu} n^{\mu}+\cdots+\alpha_{00} \quad$ This shows that we can now deal with $\psi$, the leading coefficient of which is irrational.

From now on in this section, $\varphi$ is defined by equation (2) where $\alpha_{\nu}$ is an irrational number.

We need the known identity for polynomials of degree $\nu$ :

$$
\begin{aligned}
\varphi(n+\nu) & \equiv\left(\begin{array}{l}
\nu \\
1
\end{array}\right) \varphi(n+\nu-1) \\
& -\left(\begin{array}{l}
\nu \\
2
\end{array}\right) \varphi(n+\nu-2)+\cdots+(-1)^{\nu-1}\left(\begin{array}{l}
\nu \\
\nu
\end{array}\right) \varphi(n)+\nu ! \alpha_{\nu}
\end{aligned}
$$

and the lemma:

LEMMA 1. If $F\left(x_{1}, x_{2}, \cdots, x_{\nu}\right)$ is a Riemann integrable function which is of period 1 in each variable and if $\varphi$ is a real polynomial of degree $\nu$, the leading coefficient of which is irrational, then the following equality holds:

$$
\begin{gathered}
\lim _{N \rightarrow \infty} \frac{1}{N} \sum_{n=1}^{N} F(\varphi(n), \varphi(n+1), \cdots, \varphi(n+\nu-1)) \\
=\int_{T^{\nu}} F\left(x_{1}, x_{2}, \cdots, x_{\nu}\right) d x_{1}, \cdots, d x_{\nu} .
\end{gathered}
$$


This is a very well known corollary of Weyl's theorems on uniform distribution (see for example [2]).

Combining equality (3) and Lemma 1, one can write:

$$
\begin{aligned}
& L=\lim \frac{1}{N} \sum_{n=1}^{N} \exp i \pi([\varphi(n+\nu)] \\
& \left.-\left(\begin{array}{l}
\nu \\
1
\end{array}\right)[\varphi(n+\nu-1)]+\cdots+(-1)^{\nu}\left(\begin{array}{l}
\nu \\
\nu
\end{array}\right)[\varphi(n)]\right) \\
& =\lim \frac{1}{N} \sum_{n=1}^{N} \exp i \pi\left(\left[\left(\begin{array}{l}
\nu \\
1
\end{array}\right) \varphi(n+\nu-1)\right.\right. \\
& \left.-\left(\begin{array}{l}
\nu \\
2
\end{array}\right) \varphi(n+\nu-2)+\cdots+\nu ! \alpha_{\nu}\right] \\
& \left.-\left(\begin{array}{l}
\nu \\
1
\end{array}\right)[\varphi(n+\nu-1)]+\cdots+(-1)^{\nu}[\varphi(n)]\right) \\
& =\int_{T_{\nu}} \exp i \pi\left(\left[\left(\begin{array}{l}
\nu \\
1
\end{array}\right) 2 x_{\nu}-\left(\begin{array}{l}
\nu \\
2
\end{array}\right) 2 x_{\nu-1}+\cdots+\nu ! \alpha_{\nu}\right]\right. \\
& \left.-\left(\begin{array}{l}
\nu \\
1
\end{array}\right)\left[2 x_{\nu}\right]+\cdots+(-1)^{\nu}\left[2 x_{1}\right]\right) d x_{1} \cdots d x_{\nu} .
\end{aligned}
$$

By putting $2 x_{j}=y_{j}, j=1,2, \cdots, \nu$, the integral becomes

$$
\begin{aligned}
L & =\frac{1}{2^{\nu}} \int_{(0,2)^{\nu}} \exp i \pi\left(\left[\left(\begin{array}{l}
\nu \\
1
\end{array}\right) y_{\nu}-\left(\begin{array}{l}
\nu \\
2
\end{array}\right) y_{\nu-1}+\cdots+\nu ! \alpha_{\nu}\right]\right. \\
& \left.-\left(\begin{array}{l}
\nu \\
1
\end{array}\right)\left[y_{\nu}\right]+\cdots+(-1)^{\nu}\left[y_{1}\right]\right) d y_{1} \cdots d y_{\nu} .
\end{aligned}
$$

Now the identity $[x+\varepsilon y]=[x]+\varepsilon[y]+[\{x\}+\varepsilon\{y\}], \varepsilon= \pm 1$ shows that one has:

$$
\begin{aligned}
{\left.\left[\begin{array}{l}
\nu \\
1
\end{array}\right) y_{\nu}-\left(\begin{array}{l}
\nu \\
2
\end{array}\right) y_{\nu-1}+\cdots+\nu ! \alpha_{\nu}\right]=\left(\begin{array}{l}
\nu \\
1
\end{array}\right)\left[y_{\nu}\right] } & -\left(\begin{array}{l}
\nu \\
2
\end{array}\right)\left[y_{\nu-1}\right]+\cdots+\left[\nu ! \alpha_{\nu}\right] \\
& +\left[\left(\begin{array}{l}
\nu \\
1
\end{array}\right)\left\{y_{\nu}\right\}-\cdots+\left\{\nu ! \alpha_{\nu}\right\}\right]
\end{aligned}
$$

so that:

$$
\begin{aligned}
L & = \pm \frac{1}{2^{\nu}} \int_{(0,2) \nu} \exp i \pi\left[\left(\begin{array}{l}
\nu \\
1
\end{array}\right)\left\{y_{\nu}\right\}-\left(\begin{array}{l}
\nu \\
2
\end{array}\right)\left\{y_{\nu-1}\right\}+\cdots+\left\{\nu ! \alpha_{\nu}\right\}\right] d y_{1} \cdots d y_{\nu} \\
& = \pm \int_{T_{\nu}} \exp i \pi\left[\left(\begin{array}{l}
\nu \\
1
\end{array}\right) y_{\nu}-\left(\begin{array}{l}
\nu \\
2
\end{array}\right) y_{\nu-1}+\cdots+\left\{\nu ! \alpha_{\nu}\right\}\right] d y_{1} \cdots d y_{\nu} .
\end{aligned}
$$

Consider the hyperplane $\left(\begin{array}{l}\nu \\ 1\end{array}\right) y_{\nu}-\left(\begin{array}{l}\nu \\ 2\end{array}\right) y_{\nu-1}+\cdots+(-1)^{\nu-1} y_{1}=-\left\{\nu ! \alpha_{\nu}\right\}$ in the euclidean space $\mathrm{R}^{\nu}$. It has rational coefficients except for the constant term, which is irrational. Hence it cannot split the unit cube $(0,1)^{\nu}$ into two regions of equal volume. Therefore the integral $L$ 
cannot be 0 . Finally we notice that $L$ may be written

$$
L=\lim _{N \rightarrow \infty} \frac{1}{N} \sum_{n=1}^{N} r_{n+\nu}(x)\left(r_{n+\nu-1}(x)\right)^{\left(\begin{array}{l}
(\nu) \\
1
\end{array}\right)} \cdots r_{n}(x) ;
$$

this completes the demonstration.

3. Proof of Theorem 2. Let $P$, denote the set of real polynomials of degree $\nu$, the coefficients of which are all in the interval $[0,2[$. It is easily seen that to prove Theorem 2, it is sufficient to prove the lemma:

Lemma 2. Let $E^{\nu}$ be the set of numbers $x$ such that for some $\varphi \in P_{\nu}, r_{n}(x)=\exp i \pi[\varphi(n)]$ for all integers $n$. Then the Hausdorff dimension of $E^{\nu}$ is 0 .

Let $\quad \varphi(n)=\alpha_{\nu} n^{\nu}+\cdots+\alpha_{1} n+\alpha_{0}, \quad \alpha_{j} \in[0,2]$

and let $\alpha=\left(\alpha_{0}, \alpha_{1}, \cdots, \alpha_{\nu}\right)$ be a point in the space $(0,2)^{\nu+1}$. We are going to estimate the number $N_{\nu}(p)$ of regions in $(0,2)^{\nu+1}$ which have the following property: when $\alpha$ ranges over one of these regions, the sequence $[\varphi(1)],[\varphi(2)], \cdots,[\varphi(p)]$ stays invariant. First let us show:

Lemma 3. The $h$-dimensional measure $(0 \leqq h \leqq 1)$ of the set $E_{p}^{\nu}=\left\{x \mid r_{n}(x)=\exp i \pi[\varphi(n)] ; n=1,2, \cdots, p ; \varphi \in P_{\nu}\right\}$ satisfies the inquality

$$
h \text {-meas }\left(E_{p}^{\nu}\right) \leqq \frac{N_{\perp}(p)}{2^{p_{h}}} .
$$

Indeed, when $\varphi$ runs through $P_{\nu}, \alpha$ ranges over $(0,2)^{\nu+1}$. The set $E_{p}^{\nu}$ is composed of at most $N_{\nu}(p)$ intervals, each of which has $h$-length $\left(\frac{1}{2^{p}}\right)^{h}$.

Now, if one notices that $E^{\nu}=\bigcap_{p=1}^{\infty} E_{p}^{\nu}$, one gets the result that the Hausdorff dimension of $E^{\nu}$ cannot be greater than

$$
\delta=\liminf _{p \rightarrow \infty} \frac{\log N_{\nu}(p)}{p \log 2} .
$$

We wish to show that $\delta=0$ and we shall do so by proving our last lemma : 


$$
N_{\nu}(p)=0\left(p^{(\nu+1)^{2}}\right)
$$

Proof. Let $q$ be an integer such that

$$
0 \leqq q \leqq 2\left(n^{\nu}+n^{\nu-1}+\cdots+n+1\right)-1
$$

Consider the set $R_{n, q}$ of the points $\alpha=\left(\alpha_{0}, \alpha_{1}, \cdots, \alpha_{\nu}\right)$ defined by

$$
q \leqq \alpha_{\nu} n^{\nu}+\cdots+\alpha_{1} n+\alpha_{0}<q+1 .
$$

Clearly, when $\alpha$ runs through the region $R_{n, q}$, the quantity $[\varphi(n)]=$ $\left[\alpha_{\nu} n^{\nu}+\cdots+\alpha_{1} n+\alpha_{0}\right]$ stays equal to $q$. Then let $q_{1}, q_{2}, \cdots, q_{p}$ be any sequence of integers such that $0 \leqq q_{j}<2\left(j^{\nu}+\cdots+j+1\right), j=$ $1,2, \cdots, p$. When $\alpha$ ranges over the set $\bigcap_{n=1}^{p} R_{n, q_{n}}$, the sequence $[\varphi(1)],[\varphi(2)], \cdots,[\varphi(p)]$ does not change. But the number of these regions is at most the number of different regions one can obtain by dissecting the space $\mathrm{R}^{\nu+1}$ by hyperplanes $\alpha_{\nu} n^{\nu}+\cdots+\alpha_{1} n+\alpha_{0}=q$. These hyperplanes are at most $M=M_{\nu}(p)=\sum_{j=1}^{p} 2\left(j^{\nu}+\cdots+j+1\right)=$ $0\left(p^{\nu+1}\right)$. Now, one can show that the space $\mathrm{R}^{\nu+1}$ is dissected into $0\left(M^{\nu+1}\right)$ regions by $M$ hyperplanes [5] and therefore:

$$
N_{\nu}(p)=0\left(p^{(\nu+1)^{2}}\right) \text {. }
$$

REMARK.1. It is easy to generalize Theorem 2 and obtain the following result. Let $\left(f_{n}\right)_{n \in \mathbf{N}}$ be a countable set of real functions such that

$$
\lim _{p \rightarrow \infty} \frac{\log ^{+}\left|f_{n}(p)\right|}{p}=0, \quad \forall n \in \mathbf{N} .
$$

$\left(\log ^{+}\right.$denotes the maximum of 0 and $\left.\log \right)$. Let $Q$ be the set of all real finite linear combinations of the family $\left(f_{n}\right)$. Then the Hausdorff dimension of the set $E(Q)$ is 0 .

REMARK 2. The proof of Theorem 2 shows that the set $E^{\nu}$ is not dense on the unit interval $(0,1)$. On the other hand, $E^{\nu}$ is invariant under the mapping $x \rightarrow\{2 x\}$. From these two remarks, one sees that $E^{\nu}$ is a Rajchman $H$-set and that $E(p)$ is therefore a set of uniqueness for trigonometric series. This result is to be compared with the following corollary of Pyatetski-Shapiro's theorem:

The set of nonnormal numbers is not a set of uniqueness.

\section{REFERENCES}

1. W. A. Beyer, Hausdorff dimension of level sets of some Rademacher series, Pacific J. Math. 12 (1962), 35-46.

2. J. W. S. Cassels, An Introduction to Diophantine Approximation, Cambridge University Press, 1957. 
3. J. W. S. Cassels, On a paper of Niven and Zuckerman, Pacific J. Math. 2 (1952), $555-557$.

4. M. Mendès France, Nombres normaux et fonctions pseudo-aléatoires, Ann. Inst. Fourier 13 (1963), 91-104.

5. T. S. Motzkin, The probability of solvability of linear inequalities, Proc. Second Symposium Linear Programming, Washington (1955), pp. 607-611.

6. I. Niven and H. S. Zuckerman, On the definition of normal numbers, Pacific J. Math. 1 (1951), 103-109.

University of CALifornia, Los ANGeles 


\title{
PACIFIC JOURNAL OF MATHEMATICS
}

\author{
EDITORS
}

\author{
H. SAmelson \\ Stanford University \\ Stanford, California \\ R. M. Blumenthal \\ University of Washington \\ Seattle, Washington 98105
}

\author{
J. DugundjI \\ University of Southern California \\ Los Angeles, California 90007
}

*Richard Arens

University of California

Los Angeles, California 90024

\section{ASSOCIATE EDITORS}
E. F. BECKENBACH
B. H. NeUmanN
F. WOLF
K. YoSIDA

\section{SUPPORTING INSTITUTIONS}

\author{
UNIVERSITY OF BRITISH COLUMBIA \\ CALIFORNIA INSTITUTE OF TECHNOLOGY \\ UNIVERSITY OF CALIFORNIA \\ MONTANA STATE UNIVERSITY \\ UNIVERSITY OF NEVADA \\ NEW MEXICO STATE UNIVERSITY \\ OREGON STATE UNIVERSITY \\ UNIVERSITY OF OREGON \\ OSAKA UNIVERSITY \\ UNIVERSITY OF SOUTHERN CALIFORNIA
}

\author{
STANFORD UNIVERSITY \\ UNIVERSITY OF TOKYO \\ UNIVERSITY OF UTAH \\ WASHINGTON STATE UNIVERSITY \\ UNIVERSITY OF WASHINGTON \\ * * * * \\ AMERICAN MATHEMATICAL SOCIETY \\ CALIFORNIA RESEARCH CORPORATION \\ SPACE TECHNOLOGY LABORATORIES \\ NAVAL ORDNANCE TEST STATION
}

Mathematical papers intended for publication in the Pacific Journal of Mathematics should by typewritten (double spaced). The first paragraph or two must be capable of being used separately as a synopsis of the entire paper. It should not contain references to the bibliography. No separate author's resumé is required. Manuscripts may be sent to any one of the four editors. All other communications to the editors should be addressed to the managing editor, Richard Arens, at the University of California, Los Angeles, California 90024.

50 reprints per author of each article are furnished free of charge; additional copies may be obtained at cost in multiples of 50 .

The Pacific Journal of Mathematics is published quarterly, in March, June, September, and December. Effective with Volume 13 the price per volume (4 numbers) is $\$ 18.00$; single issues, $\$ 5.00$. Special price for current issues to individual faculty members of supporting institutions and to individual members of the American Mathematical Society: $\$ 8.00$ per volume; single issues $\$ 2.50$. Back numbers are available.

Subscriptions, orders for back numbers, and changes of address should be sent to Pacific Journal of Mathematics, 103 Highland Boulevard, Berkeley 8, California.

Printed at Kokusai Bunken Insatsusha (International Academic Printing Co., Ltd.), No. 6, 2-chome, Fujimi-cho, Chiyoda-ku, Tokyo, Japan.

PUBLISHED BY PACIFIC JOURNAL OF MATHEMATICS, A NON-PROFIT CORPORATION

The Supporting Institutions listed above contribute to the cost of publication of this Journal, but they are not owners or publishers and have no responsibility for its content or policies.

* Basil Gordon, Acting Managing Editor until February 1, 1966. 


\section{Pacific Journal of Mathematics}

\section{Vol. 15, No. $4 \quad$ December, 1965}

Robert James Blattner, Group extension representations and the structure space ........... 1101

Glen Eugene Bredon, On the continuous image of a singular chain complex .............. 1115

David Hilding Carlson, On real eigenvalues of complex matrices .................... 1119

Hsin Chu, Fixed points in a transformation group ............................. 1131

Howard Benton Curtis, Jr., The uniformizing function for certain simply connected Riemann

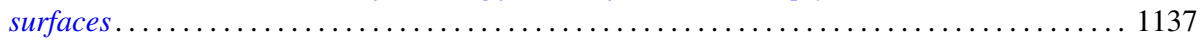

George Wesley Day, Free complete extensions of Boolean algebras................... 1145

Edward George Effros, The Borel space of von Neumann algebras on a separable Hilbert

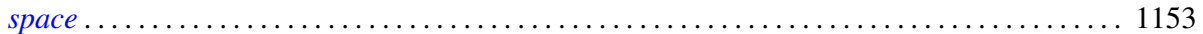

Michel Mendès France, $A$ set of nonnormal numbers ......................... 1165

Jack L. Goldberg, Polynomials orthogonal over a denumerable set ................ 1171

Frederick Paul Greenleaf, Norm decreasing homomorphisms of group algebras . . . . . . . . 1187

Fletcher Gross, The 2-length of a finite solvable group ........................ 1221

Kenneth Myron Hoffman and Arlan Bruce Ramsay, Algebras of bounded sequences ........ 1239

James Patrick Jans, Some aspects of torsion . . . . . . . . . . . . . . . . . . . . . . . 1249

Laura Ketchum Kodama, Boundary measures of analytic differentials and uniform

approximation on a Riemann surface ............................... 1261

Alan G. Konheim and Benjamin Weiss, Functions which operate on characteristic

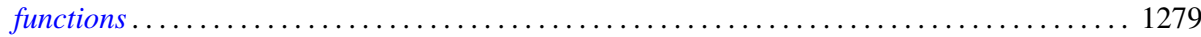

Ronald John Larsen, Almost invariant measures ............................ 1295

You-Feng Lin, Generalized character semigroups: The Schwarz decomposition ............ 1307

Justin Thomas Lloyd, Representations of lattice-ordered groups having a basis . . . . . . . . 1313

Thomas Graham McLaughlin, On relative coimmunity ....................... 1319

Mitsuru Nakai, $\Phi$-bounded harmonic functions and classification of Riemann surfaces ....... 1329

L. G. Novoa, On n-ordered sets and order completeness ..................... 1337

Fredos Papangelou, Some considerations on convergence in abelian lattice-groups . . . . . . . 1347

Frank Albert Raymond, Some remarks on the coefficients used in the theory of homology

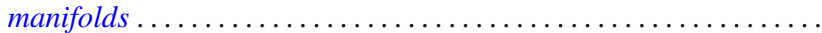

John R. Ringrose, On sub-algebras of a $C^{*}$-algebra .

Jack Max Robertson, Some topological properties of certain spaces of differentiable

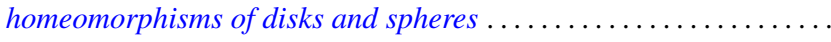

Zalman Rubinstein, Some results in the location of zeros of polynomials

Arthur Argyle Sagle, On simple algebras obtained from homogeneous general Lie triple systems. . . .

Hans Samelson, On small maps of manifolds ............................... 1401

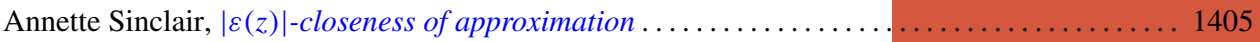

Edsel Ford Stiel, Isometric immersions of manifolds of nonnegative constant sectional curvature

Earl J. Taft, Invariant splitting in Jordan and alternative algebras ................. 1421

L. E. Ward, On a conjecture of R. J. Koch . . . . . . . . . . . . . . . . . . . . . . . . . . . 1429

Neil Marchand Wigley, Development of the mapping function at a corner . . . . . . . . . . 1435

Horace C. Wiser, Embedding a circle of trees in the plane ....................... 1463

Adil Mohamed Yaqub, Ring-logics and residue class rings . . . . . . . . . . . . . . . . 1465

John W. Lamperti and Patrick Colonel Suppes, Correction to: Chains of infinite order and their application to learning theory ........................................ 1471

Charles Vernon Coffman, Correction to: Non-linear differential equations on cones in Banach

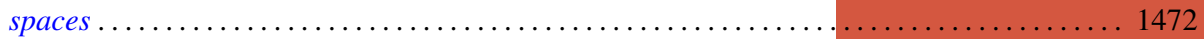

P. H. Doyle, III, Correction to: A sufficient condition that an $\operatorname{arc}$ in $S^{n}$ be cellular. . . . . . . . 1474

P. P. Saworotnow, Correction to: On continuity of multiplication in a complemented algebra 